\title{
Collisional rates for vib-rotational transitions in diatomic molecules ${ }^{\star}$
}

\begin{abstract}
S. Chandra ${ }^{1, \star \star}$ and A. K. Sharma ${ }^{2}$
1 School of Physical Sciences, Swami Ramanand Teerth Marathwada University, Nanded 431 606, India

2 B.R.C.M. College of Engineering and Technology, Bahal 127 028, India

Received 19 May 1999 / Accepted 28 May 2001

Abstract. A number of diatomic molecules have been found in vibrationally excited states in several cosmic objects. The molecules in vibrationally excited states provide valuable information about the physical conditions prevailing near star forming regions and in circumstellar envelopes of late-type stars. To analyze the spectrum of such molecules, some of the important parameters required are the collisional rate coefficients for vib-rotational transitions in the molecule. Currently, knowledge of collisional rate coefficients is very poor. Here, we discuss a method for calculating the collisional rate coefficients for vib-rotational transitions in a diatomic molecule, where the colliding partner $\mathrm{H}_{2}$ is considered as a structureless particle. This method is quite good for high temperatures (usually found in star-forming regions and in circumstellar envelopes of late-type stars), but may be questioned for low temperatures. As an example, calculations for the CS molecule at $500 \mathrm{~K}$ for three vibrational states are presented.
\end{abstract}

Key words. molecular data - stars: circumstellar matter

\section{Introduction}

A number of diatomic molecules, observed in several cosmic objects, have been found in vibrationally excited states. Examples of such molecules are CS, CO, SiO. To be vibrationally excited, the molecule must be in an unusually hot and dense gas, and/or in a region where a strong IR radiation field is present. Hence, vibrationally excited molecules may play an important role as a probe for finding out the physical conditions near star-forming regions and in circumstellar envelopes of late type stars.

To analyze the spectrum from a molecule observed in vibrationally excited states, one requires the knowledge of the rate coefficients for radiative and collisional transitions between the rotational levels lying in various vibrational states (i.e., for vib-rotational transitions). Einstein Acoefficients for vib-rotational (including pure rotational) transitions for the CS and $\mathrm{CO}$ molecules have been calculated by Chandra et al. (1995, 1996), respectively.

Currently, knowledge of transition rate coefficients for collision of a molecule with hydrogen molecule is

Send offprint requests to: S. Chandra,

e-mail: chandrasuresh@123india.com

* Tables 1 and 3 are only available in electronic form at the CDS via anonymous ftp to cdsarc.u-strasbg.fr (130.79.128.5) or via

http://cdsweb.u-strasbg.fr/cgi-bin/qcat?J/A+A/376/356

$\star \star$ Visiting Associate of the Inter-University Centre for Astronomy \& Astrophysics, Pune 411 007, India. very poor. Collisional rate coefficients for a limited number of rotational transitions for some molecules have been reported in the literature. For example, data for $\mathrm{H}_{2}-\mathrm{H}_{2}$ (Green et al. 1978), $\mathrm{H}_{2}$-CS (Green \& Chapman 1978) and $\mathrm{H}_{2}$ - $\mathrm{CO}$ (Green \& Thaddeus 1976) systems are available.

In the present investigation, we have discussed a method by which collisional rate coefficients for vibrotational (including pure rotational) transitions may be calculated. Here, the colliding partner $\mathrm{H}_{2}$ is considered as a structureless particle. This method is valid at high temperatures (say, above $300 \mathrm{~K}$ ), but may be questioned at low temperatures. The regions where the knowledge of vib-rotational transitions is required, however, are at high temperature.

It is always advisable to calculate collisional rate coefficients for one direction (either downward or upward), and then to calculate the rate coefficients in the opposite direction within the computer programme where these rate coefficients are to be used, with the help of the detailed equilibrium equation. Here, we have discussed a method for the rate coefficients in the downward (called de-excitation) direction.

\section{Method for calculation of collisional rate coefficients}

In a cosmic object, the molecule under investigation is considered to collide with $\mathrm{H}_{2}$ molecules only, as these 
collisions are likely to be dominant because of the large abundance of $\mathrm{H}_{2}$. In the molecule, we have two types of transitions: (i) pure rotational transitions within a vibrational state, and (ii) transitions between rotational levels in different vibrational states. Within a vibrational state, the rate coefficients for the rotational de-excitation transitions, connected to the ground rotational level, may be calculated with the help of the relation

$$
\begin{aligned}
& C(T \mid J \rightarrow 0, \Delta v=0)=\frac{K_{v}(T) T^{-1 / 2}}{2 J+1} \\
& \times\left[1-\exp \left(k T / h \nu_{J 0}\right)\right],
\end{aligned}
$$

where $h$ is the Planck constant, $k$ the Boltzmann constant, $J$ the rotational quantum number, $v$ the vibrational quantum number, $\nu_{J 0}$ the frequency for the transition, and $K_{v}(T)$ the constant depending on $v$ and the kinetic temperature $T$. This expression reproduces the limiting behaviour, at high and low temperatures, as suggested by Goldreich \& Kwan (1974).

For the intermediate, level-to-level de-excitations, the rate coefficients may be calculated by using the relation

$$
\begin{aligned}
& C\left(T \mid J_{\mathrm{u}} \rightarrow J_{\mathrm{l}}, \Delta v=0\right)=\left(2 J_{\mathrm{l}}+1\right) \sum_{J=\left|J_{\mathrm{u}}-J_{1}\right|}^{J_{\mathrm{u}}+J_{1}}\left(\begin{array}{ccc}
J_{\mathrm{u}} & J_{\mathrm{l}} & J \\
0 & 0 & 0
\end{array}\right)^{2} \\
& \times(2 J+1) C(T \mid J \rightarrow 0, \Delta v=0), \quad(2)
\end{aligned}
$$

where ( ) is a $3-j$ symbol. The suffices $u$ and $l$ are used for the upper and lower energy levels, respectively.

For the individual level-to-level transitions, connecting the two vibrational states, the rate coefficients may be assumed to be proportional to the rate coefficients for the corresponding rotational transitions in the ground vibrational state, such that

$C\left(T \mid v, J_{\mathrm{i}} \rightarrow v^{\prime}, J_{\mathrm{f}}\right)=P_{v v^{\prime}} C\left(T \mid 0, J_{\mathrm{i}} \rightarrow 0, J_{\mathrm{f}}\right)$

where $P_{v v^{\prime}}$ is a dimensionless, symmetrical parameter, characterizing the two vibrational states, and is given by

$P_{v v^{\prime}}=N_{\mathrm{t}} C\left(T \mid v \rightarrow v^{\prime}\right) / \sum_{J_{\mathrm{i}} J_{\mathrm{f}}} C\left(T \mid 0, J_{\mathrm{i}} \rightarrow 0, J_{\mathrm{f}}\right)$,

where $C\left(T \mid v \rightarrow v^{\prime}\right)$ is the de-excitation rate coefficient for the transition from the upper vibrational state $v$ to the lower vibrational state $v^{\prime}$, and $N_{\mathrm{t}}$ the number of the transitions accounted for in the summation. Equation (3), however, decouples vibrational and rotational transitions and is a rather poor approximation. Here, we would not be able to calculate the rate coefficients for the vib-rotational transitions for which $\Delta J=0$. However, the absence of these rate coefficients would not affect the investigation significantly. The rate coefficients for the vibrational transition $1 \rightarrow 0$ are given by (Lifshitz 1974; Chandra 1994)

$$
C(T \mid 1 \rightarrow 0)=\frac{R T / P \tau}{1-\mathrm{e}^{\theta / T}}
$$

where

$P \tau=\exp \left[A\left(T^{-1 / 3}-0.015 \mu^{1 / 4}\right)-18.42\right]$,

$\theta=1.44\left(\omega_{\mathrm{e}}-2 \omega_{\mathrm{e}} x_{\mathrm{e}}\right)$,

$A=1.16 \times 10^{-3} \theta^{4 / 3} \mu^{1 / 2}$.

Here, $\mu$ is the reduced mass of the colliding system, $\omega_{\mathrm{e}}$ and $\omega_{\mathrm{e}} x_{\mathrm{e}}$ the physical parameters for the molecule, $R$ the gas constant, and $\theta$ the characteristic temperature of the molecule. Equation (5) is obtained by a parameterization of measured vibrational relaxation times for several diatomic molecules for temperatures higher than $300 \mathrm{~K}$. Thus, the use of Eq. (5) for temperatures less than $300 \mathrm{~K}$ may be highly questionable. The rate coefficient for the transitions between the adjacent vibrational states can be obtained using the relation (Procaccia \& Levine 1975)

$$
\begin{aligned}
C\left(T \mid v \rightarrow v^{\prime}\right)= & C(T \mid 1 \rightarrow 0)[(v+1) \\
& \left.\times \exp \left(-h c \omega_{\mathrm{e}} / k T\right) \delta_{v^{\prime}-1, v}+v \delta_{v^{\prime}+1, v}\right]
\end{aligned}
$$

where $\delta$ is the Kronecker delta. Further, we know (Procaccia \& Levine 1975)

$C(T \mid v)=\sum_{v^{\prime}} C\left(T \mid v \rightarrow v^{\prime}\right)$

and may assume that

$\frac{C\left(T \mid v \rightarrow v_{1}\right)}{C\left(T \mid v \rightarrow v_{2}\right)}=\frac{2 v_{1}+1}{2 v_{2}+1}$.

The validity of the aforesaid method is quite good for the large kinetic temperature $T$. It may, however, be questioned for low temperature. In the absence of any other, better solution, the present method may be used for calculating the rate coefficients.

\subsection{Detailed equilibrium equation}

Once the de-excitation rate coefficients are known, the corresponding excitation rate coefficients can be calculated by using the equation of the detailed balance.

$$
\begin{gathered}
C\left(v_{\mathrm{l}}, J_{\mathrm{l}} \rightarrow v_{\mathrm{u}}, J_{\mathrm{u}}\right)=\frac{2 J_{\mathrm{u}}+1}{2 J_{\mathrm{l}}+1} C\left(v_{\mathrm{u}}, J_{\mathrm{u}} \rightarrow v_{\mathrm{l}}, J_{\mathrm{l}}\right) \\
\quad \times \exp \left[-\frac{h c\left\{E\left(v_{\mathrm{u}}, J_{\mathrm{u}}\right)-E\left(v_{\mathrm{l}}, J_{\mathrm{l}}\right)\right\}}{k T}\right],
\end{gathered}
$$

where $c$ is the speed of light. The energy $E$ of the levels is in $\mathrm{cm}^{-1}$.

\section{Collisional rate coefficients for the CS molecule}

As an example, let us consider the case of the ${ }^{12} \mathrm{C}^{32} \mathrm{~S}$ molecule colliding with structureless $\mathrm{H}_{2}$ molecules. Here, we accounted for the three vibrational states, $v=0,1$ and 2 only of the CS molecule, and thus from Eqs. (6) through (8), we have

$$
C(T \mid 2 \rightarrow 1)=2 C(T \mid 1 \rightarrow 0)
$$


and

$C(T \mid 2 \rightarrow 0)=\frac{1}{3} C(T \mid 2 \rightarrow 1)=\frac{2}{3} C(T \mid 1 \rightarrow 0)$.

Now, we have the relations

$$
P_{21}=2 P_{10} \quad \text { and } \quad P_{20}=\frac{2}{3} P_{10} .
$$

In these vibrational states, we considered only those rotational levels which lie below the next higher vibrational state, and hence, we have accounted for rotational levels up to $J=39,38$, and 38 , in the vibrational states, $v=0$, 1 , and 2 , respectively. Thus, in all, our system is consists of 118 energy levels. Energy, along with the statistical weight, vibrational quantum number, and the rotational quantum number of the levels are given in Table 1, which is available in electronic form via anonymous ftp. In the present investigation, we used the same value for $K_{v}(T)$ for all three vibrational states, i.e., $K_{v}(T)=K_{0}(T)$. The value of $K_{0}(T)$ for the kinetic temperature $500 \mathrm{~K}$ is taken as $1 \times 10^{-9} \mathrm{~cm}^{3} \mathrm{~s}^{-1} \mathrm{~K}^{1 / 2}$.

The value of $K_{0}(T)$ varies with the kinetic temperature. For some kinetic temperatures, we have given the value of $P_{10} K_{0}(T)$ in Table 2 .

Table 2. Value of $P_{10} K_{0}(T)$ at various temperatures

\begin{tabular}{|c|c|}
\hline $\begin{array}{c}\text { Temp } \\
(\mathrm{K})\end{array}$ & $\begin{array}{c}P_{10} K_{0}(T) \\
\left(\mathrm{cm}^{3} \mathrm{~s}^{-1} \mathrm{~K}^{1 / 2}\right)\end{array}$ \\
\hline 500 & $8.467 \times 10^{-12}$ \\
1000 & $5.195 \times 10^{-11}$ \\
1500 & $1.542 \times 10^{-10}$ \\
2000 & $3.401 \times 10^{-10}$ \\
2500 & $6.349 \times 10^{-10}$ \\
\hline
\end{tabular}

It is found that at low temperatures, the rates for vibrotational transitions are not significant in comparison to those for the pure rotational transitions. However, they are essential as they connect the vibrational states. But, at high temperatures, the rates for the vib-rotational transitions are quite significant.

Collisional rate coefficients for the downward transitions at $500 \mathrm{~K}$ are calculated, and are given in Table 3 in electronic form via anonymous ftp.

For the values for collisional rate coefficients for vibrotational transitions for other higher temperatures, and for other molecules, readers may contact the authors.

Acknowledgements. We are thankful to Prof. Dr. W. H. Kegel of the university of Frankfurt/Main, Germany for his encouragement. A part of the work was completed in Frankfurt during the visit of Prof. Chandra under the scientific exchange programme between the INSA, New Delhi, India and the DFG, Bonn, Germany.

\section{References}

Chandra, S. 1994, Ap\&SS, 218, 29

Chandra, S., Kegel, W. H., Le Roy, R. J., \& Hertenstein, T. 1995, A\&AS, 114, 175

Chandra, S., Maheshwari, U. V., \& Sharma, A. K. 1996, A\&AS, 117,557

Goldreich, P., \& Kwan, J. 1974, ApJ, 189, 441

Green, S., \& Chapman, S. 1978, ApJS, 37, 169

Green, S., Ramaswamy, R., \& Rabitz, H. 1978, ApJS, 36, 483

Green, S., \& Thaddeus, P. 1976, ApJ, 205, 766

Lifshitz, A. 1974, J. Chem. Phys., 61, 2478

Procaccia, I., \& Levine, R. D. 1975, J. Chem. Phys., 63, 4261 